vulnerabilities related to age, socioeconomic status, life course factors, and engagement in high risk behaviour such as sex trade. We present a risk factor analysis for those with single and multiple STIs, using the Enhanced Surveillance of Canadian Street Youth (E-SYS) data.

Methods E-SYS is a cross-sectional surveillance system of streetinvolved youth (15-24 years). Participants from seven urban centres completed an interviewer-administered questionnaire and were tested for multiple sexually transmitted and blood-borne infections. For this analysis, data were restricted to five infections (HIV, chlamydia, gonorrhoea, syphilis and HSV-2). Data from three cycles (2001-2006) were analysed to determine total number of infections per participant using an iterative tracker. Participants who indicated previous participation were excluded from the analysis to avoid double-representation.

Results Of those who provided biological specimens ( $\mathrm{n}=3823)$, $17.0 \%$ tested positive for one infection and $3.4 \%$ tested positive for two or more infections (Abstract O1-S04.03 table 1). Over half (15 of 28) of HIV-positive individuals were multiply-infected. Risk factors for multiple infections included older age $(p=0.0449)$, being Aboriginal ( $p=0.0061$; particularly females), being HIV infected $(p=<0.0001)$, having a previous history of an STI $(p=0.0144)$, pregnancy $(p=0.0079)$, and reporting prostitution as the primary source of income $(p=0.0028)$.

Abstract 01-S04.03 Table 1 Single and multiple STI in the enhanced street youth surveillance population

\begin{tabular}{llllll}
\hline & Chlamydia & Gonorrhoea & Syphilis & HSV-2 & HIV \\
\hline Chlamydia & $268(7.98 \%)$ & & & & \\
Gonorrhoea & $42(1.26 \%)$ & $17(2.35 \%)$ & & & \\
Syphilis & $2(0.07 \%)$ & $2(0.07 \%)$ & $3(0.09 \%)$ & & \\
HSV2 & $72(2.78 \%)$ & $19(0.74 \%)$ & $4(0.14 \%)$ & $348(12.20 \%)$ & \\
HIV & $4(0.15 \%)$ & $0(0.00 \%)$ & $1(0.03 \%)$ & $14(0.50 \%)$ & $13(0.44 \%)$ \\
Tested overall & 3357 & 3339 & 3190 & 2852 & 2951 \\
Total \# of individuals & 375 & 69 & 10 & 442 & 28 \\
infected & & & & & \\
Prevalence overall & $11.17 \%$ & $2.07 \%$ & $0.56 \%$ & $15.50 \%$ & $0.95 \%$ \\
\hline
\end{tabular}

${ }^{*}$ Same-infection crosses represent single infections.

$\%$ 's based on individuals tested for both infections.

An individual may be counted more than once if they have more than two infections.

Conclusions The prevalence of certain STI co-infections (eg, chlamydia and gonorrhoea) is high among street-involved youth and within this population, certain sub-groups may be more vulnerable to these co-infections. Continued efforts are required to promote comprehensive STI testing among street-involved youth and to raise awareness of the potential for multiple infections.

\section{1-S04.04 PREDICTORS OF REPEAT CHLAMYDIA TRACHOMATIS AND/OR NEISSERIA GONORRHOEAE INFECTIONS AMONG AFRICAN-AMERICAN ADOLESCENT FEMALES}

doi:10.1136/sextrans-2011-050109.22

A Swartzendruber, R DiClemente, J Sales, J L Brown, E Rose. Emory University Rollins School of Public Health, Atlanta, USA

Background Young African-American females have the highest rates of Chlamydia (CT) and gonorrhoea (GC) in the US. Few studies have explored predictors of repeat STDs among this population. The objective was to identify predictors of repeat CT and/or GC infections among African-American adolescent females positive for at least one of these STDs at baseline.

Methods Sociodemographic, psychosocial and sexual behaviour data were collected via ACASI at baseline and every 6 months for 2 years from 701 African-American females (15-19 years) enrolled in an
HIV prevention trial. Vaginal swabs were self-collected at each visit and assayed for CT and GC using DNA amplification. Participants with a positive test result received directly observable single-dose antimicrobial treatment and risk-reduction counselling. Repeat infection was defined as a positive test result following a negative result or documented treatment. Among those who tested positive for CT and/or GC at baseline, bivariate and multivariable analyses compared study variables for those who did vs did not have a repeat infection during the 2-year follow-up.

Results Of 615 (88\%) participants with $\geq 1$ follow-up test result, 122 (20\%) had a positive CT and/or GC test result at baseline; 49 (40\% of baseline positives and $8 \%$ of total) had a repeat infection during the study period. Of those with a repeat infection, 30 (61\%) were positive at only one follow-up visit, $18(37 \%)$ at two and $1(2 \%)$ at three follow-up visits. In bivariate analyses, relative to those positive at baseline only, participants with repeat infection were less likely to have a boyfriend ( 71 vs $83 \%, p=0.043$ ) and see themselves marrying their current boyfriend ( 57.1 vs $76.2 \%, p=0.05$ ) and more likely to have had sex with a man who had sex with other men $(8.2$ vs $0 \%$, $\mathrm{p}=0.013$ ). Participants with repeat infection had higher mean impulsivity scores $(p=0.027)$. Controlling for age and treatment assignment, greater impulsivity (AOR: 1.1, $\mathrm{p}=0.018$ ) was associated with increased likelihood of a repeat infection and having a boyfriend (AOR: $0.21, p=0.006$ ) with decreased likelihood of a repeat infection. Conclusions Repeat CT and/or GC infections are common among African-American adolescent females. Among young African-American females who test positive for CT and/or GC, tailored interventions for more impulsive adolescents and those without a boyfriend may help prevent repeat infections.

\section{1-S04.05 INCIDENCE AND PREVALENCE OF SEXUALLY TRANSMITTED INFECTIONS AMONG SCHOOL STUDENTS IN THE EASTERN CAPE, SOUTH AFRICA}

doi:10.1136/sextrans-2011-050109.23

${ }^{1} \mathrm{~F}$ Radebe, ${ }^{2} \mathrm{~J}$ Jemmott III, ${ }^{3} \mathrm{~A}$ Klopper, ${ }^{2} \mathrm{~L}$ Jemmott, ${ }^{4} \mathrm{~A}$ O'Leary, ${ }^{5} \mathrm{Z}$ Ngwane, ${ }^{2} \mathrm{C}$ Carty, ${ }^{6} \mathrm{~J}$ Tyler, ${ }^{1} \mathrm{~V}$ Maseko, ${ }^{1} \mathrm{D}$ Lewis. ${ }^{1}$ National Institute for Communicable Diseases, National Health Laboratory Service, Sandringham, South Africa; ${ }^{2}$ University of Pennsylvania, Philadelphia, USA; ${ }^{3}$ Private General Practice East London, South Africa; ${ }^{4}$ Centers for Disease Control and Prevention, Atlanta, USA; ${ }^{5}$ Haverford College, Haverford, USA; ${ }^{6}$ University of Fort Hare, Alice, South Africa

Background Although STIs are an important co-factor for HIV acquisition, few community-based STI prevalence/incidence data exist for youth in South Africa.

Methods School students ( $\mathrm{n}=1057)$, enrolled in a cluster-randomised intervention trial in the Eastern Cape, were tested for STIs at the 42 (42M) and 54 (54M) month follow-up visits. Students filled in questionnaires, provided blood for herpes simplex type 2 (HSV-2) serology (HerpeSelect IgG, Focus Diagnostics) and urine to test for Neisseria gonorrhoeae (NG), Chlamydia trachomatis (CT) and Trichomonas vaginalis (TV) (Aptima Combo 2 and Aptima TV, GenProbe). Positive NG/CT/TV results were confirmed by other assays (Aptima NG, Aptima CT, Gen-Probe; T vaginalis Real-TM, Sacace Biotechnologies). Students with NG/CT/TV received treatment and partner follow-up; those with HSV-2 infection were counselled. STI prevalence was determined by gender at each visit; overall incidence was estimated using results for all students attending both visits and also for a subgroup who reported ever having had vaginal intercourse. Descriptive statistical analysis was performed and associations investigated with the $\chi^{2}$ test.

Results 959 (91\%) and 977 (92\%) students tested for STIs at 42M and 54M, respectively. The students' mean age (SD) was 15.8 (1.25) years at $42 \mathrm{M}$ and $16.8(1.25)$ years at $54 \mathrm{M}$. At $42 \mathrm{M}, 149(15.5 \%)$ had curable STIs and $67(7.0 \%)$ had HSV-2 infection. At 54M, 154 (15.8\%) had curable STIs and 104 (10.7\%) had HSV-2 infection. All 